\title{
$\alpha$ Centauri revisited: when the spectroscopic and the astrometric mass ratios become consistent
}

\section{Pourbaix}

Royal Observatory of Belgium, Avenue circulaire 3, B-1180 Bruxelles, Belgium, e-mail: pourbaix@astro.ulb.ac.be

\begin{abstract}
Precise radial velocities lately published (Murdoch, Hearnshaw \& Clark 1993) allow a simultaneous least-squares adjustment of all visual and spectroscopic observations. Starting with the same data as Murdoch \& Hearnshaw (1993), we obtain a mass ratio that agrees better with Kamper \& Wesselink's (1978) astrometric estimate. Our results suggest upward revisions of the distance to the system as well as of the individual masses of the components.
\end{abstract}

\section{Introduction}

$\alpha$ Cen is the binary system closest to Earth and one could think it has been studied so intensively that there is nothing new to discover. It is true that it has been visually measured for almost 250 years but its long period' coupled to the poor quality of the spectroscopic measurements, leads to a surprisingly large remaining uncertainty on the individual masses. Precise radial velocities lately published (Murdoch, Hearnshaw, \& Clark 1993) have radically changed the situation. Coupling them to visual observations, one derives a parallax leading to a significant upward revision of the individual masses with respect to previous determinations.

\section{First orbit determinations}

Downing (Glaisher \& Knobel 1884) originally suggested that to undertake spectroscopic observations of $\alpha$ Cen might be useful. Actually, it took twenty years before Wright (1904) first reported his measurements of the individual radial velocities of both components. Using the three velocities available for each component and the visual solution by Roberts $(1893,1895)$, Wright obtained $760 \pm 30$ mas for the parallax and a mass sum of $1.9 \pm 0.2 M_{\odot}$.

Though visual orbits date back to last century (Roberts 1893, 1895), almost three revolutions had taken place after the first visual observation before Heintz (1958) proposed the first reliable visual orbit, based on photographic plates only. Owing to the relative motion of the system with respect to the Sun and owing also to the short distance to the system, a secular variation of some orbital parameters might be noticeable. Heintz introduced that variation through a first-order correction of the four angular terms. He has lately confirmed his initial orbit (Heintz 1982). Although 28 new observations have appeared between 1958 
and 1981 , he has not taken them into account to refine his 1958 orbit. In spite of that, his solution is still quoted as the reference par excellence (Demarque, Guenther, \& van Altena 1986).

\section{Spectroscopic and astrometric mass ratios}

Using former spectra (Lunt 1918, Campbell \& Moore 1928, Jones 1928) and his own new ones, Wesselink (1953) computed the first mass ratio based on spectroscopic data, $M_{B} / M_{A}=0.4$, which is really discrepant with the 0.82 coming from meridian observations (Boss General Catalogue). The fairly good precision of Wesselink's measurements coupled with the fact that they are still the only radial velocities close to the extrema makes them very useful.

Nowadays, the mass ratio the most trusted is Kamper \& Wesselink's (1978). From all photographic plates taken in different observatories up to 1971 , they derived $\kappa=0.454 \pm 0.002\left(\kappa \equiv M_{B} /\left(M_{A}+M_{B}\right)\right)$ and $\varpi=750$ mas. These values combined with Heintz' (1958) orbit yield $M_{A}=1.10 M_{\odot}$ and $M_{B}=0.91 M_{\odot}$.

Recently, Murdoch et al. (1993) obtained very precise radial velocity measurements of both components. Though only three years are covered by the observations, they are precise enough to estimate a reliable spectroscopic mass ratio. Murdoch \& Hearnshaw (1993) used these data to determine $M_{B} / M_{A}=$ $0.75 \pm 0.09$, just at the limit of consistency with Kamper \& Wesselink's (1978) solution.

To derive that mass ratio, Murdoch \& Hearnshaw (1993) fitted the pairs $\left(V_{B}, V_{A}\right)$ with the ordinary least-squares bisector (Isobe et al. 1990). The main drawbacks of this procedure are its sensitivity to outliers and to the fitted straight line (when the segment covered by the observations is small). The radial velocities of Murdoch et al. (1993) have both weaknesses; there are some outliers and the observations only cover three per cent of the orbit.

\section{The parallax}

Jones (1928) computed the orbital parallax of the system based on his own radial velocities taken at the Cape between 1908 and 1924 as well as on Lohse's (1910) visual orbit. He obtained $\varpi=767$ mas, close to Wright's (1904) first spectroscopic estimate. Using a preliminary visual orbit by Hertzsprung ${ }^{1}$ and all existing radial velocities, Wesselink (1953) derived $776 \pm 8$ mas, close to Wright's also.

Kamper \& Wesselink (1978) undertook a new reduction of almost 300 photographic plates coming mostly from the Cape and from Johannesburg. They obtained $750 \pm 5$ mas as a weighted mean of their different parallaxes. Demarque et al. (1986) slightly revised that result. By changing the weights associated to the different sources of parallaxes, their new solution is $\varpi=750.6 \pm 4.6$ mas.

\footnotetext{
${ }^{1}$ the reference is not quoted in Wesselink's (1953) article
} 


\section{Simultaneous spectro-visual adjụstment}

Up to now, there has been no simultaneous adjustment of the visual and spectroscopic data. From Batten, Fletcher \& MacCarthy (1989), one could even believe that there has been no spectroscopic orbit since Lunt's (1918).

Unlike Heintz (1958), we simultaneously fit (Pourbaix 1998) all visual observations, photographic as well as micrometric, in a least-squares adjustment. These 415 points are distributed almost uniformly over three revolutions. As shown on Figure 1, we do not confirm the remark by Heintz (1982) about the substantial over-estimate of the separation in micrometric observations. The radial velocities of Murdoch et al. (1993) are the most precise and accurate(?) and can thus be used as the initial set of radial velocities.

Table 1. Orbital parameters and their standard deviations of $\alpha$ Cen. Columns 2 and 3 result from the simultaneous adjustment of all visual observations and the radial velocities of Murdoch et al. (1993). For columns 4 and 5 , all spectroscopic and visual data have been used

\begin{tabular}{lcccc}
\hline Element & Value & Std. dev. & Value & Std. dev. \\
\hline$a\left(^{\prime \prime}\right)$ & 17.59 & 0.028 & 17.59 & 0.028 \\
$i\left(^{\circ}\right)$ & 79.23 & 0.046 & 79.23 & 0.046 \\
$\omega\left(^{\circ}\right)$ & 231.8 & 0.15 & 231.8 & 0.15 \\
$\Omega\left(^{\circ}\right)$ & 204.82 & 0.087 & 204.82 & 0.087 \\
$e$ & 0.519 & 0.0013 & 0.519 & 0.0013 \\
$P(\mathrm{yr})$ & 79.90 & 0.013 & 79.90 & 0.013 \\
$T($ Besselian year $)$ & 1955.59 & 0.019 & 1955.59 & 0.019 \\
$V_{0}(\mathrm{~km} / \mathrm{s})$ & -21.9 & 0.15 & -21.87 & 0.054 \\
$\varpi(\mathrm{mas})$ & 737.0 & 2.6 & 737.0 & 2.6 \\
$\kappa$ & 0.45 & 0.036 & 0.45 & 0.013 \\
mass A $\left(M_{\odot}\right)$ & 1.2 & 0.078 & 1.16 & 0.031 \\
mass B $\left(M_{\odot}\right)$ & 1.0 & 0.076 & 0.97 & 0.032 \\
\hline \hline
\end{tabular}

The resulting orbit is given in columns 2 and 3 of Table 1 . All parameters constrained by the visual observations are characterized by small standard deviations, consistent with the large number of data. Things are different for $V_{0}$ and $\kappa$. They are both poorly constrained as their standard deviations indicate. That is also confirmed by the +0.99-correlation between these two parameters.

The confidence interval of $\kappa$ is rather large but the central value is in excellent agreement with the astrometric result (Kamper \& Wesselink 1978). So by using a more suitable fitting tool (or function) than Murdoch \& Hearnshaw (1993), one can recover Kamper \& Wesselink's result from the radial velocities of Murdoch et al. (1993). The precision of the radial velocities is as good as announced by Murdoch et al.: $42 \mathrm{~m} / \mathrm{s}$ for $\mathrm{A}$ and $76 \mathrm{~m} / \mathrm{s}$ for B, both well under the $100 \mathrm{~m} / \mathrm{s}$ announced in the paper.

Since the previous result confirms the astrometric mass ratio, we can sequentially add new data sets, trying to keep that ratio as close as possible to the current result. When a new group of radial velocities is added, one allows the 
overall group to be shifted in such a manner that the mean of the residuals from both components is null. No differential correction is applied to one component with respect to the other.

Here is the list of groups coupled to the data of Murdoch et al. (1993), to compose the final radial velocity set:

- Hough (1911) and Jones (1928) published two sets of radial velocities taken at the Cape Observatory in the periods 1904-1908 and 1908-1924.

- Lunt (1918) took some spectra between 1904 and 1912.

- Campbell \& Moore (1928) compiled the radial velocities obtained at Santiago between 1904 and 1922. The authors gave empirical corrections to apply to individual measurements depending on who obtained them, where and when.

- Wesselink (1953) took some spectra around the extrema of the radial velocities. Unfortunately, though the epoch of these extrema had been known for a long time (Jones 1928), very few spectra were taken at that epoch.

- Archer (1957) obtained some points in the steepest part of the radial velocity curves, just after the extrema.

- Jones \& Fisher (1984) determined just one radial velocity of each component in 1972 which almost coincides with the secondary extrema.

The shift applied to each set as well as the standard deviations of the radial velocities within each group are summarized in Table 2.

Table 2. Shift and standard deviations for each group of radial velocities. $\left({ }^{*}\right)$ Wright's (1904) measurements are not included. $\left({ }^{* *}\right)$ Since there is only one observation, these $\sigma$ are just the residuals.

\begin{tabular}{lccc}
\hline Reference & Shift $(\mathrm{km} / \mathrm{s})$ & $\sigma_{V_{A}}(\mathrm{~km} / \mathrm{s})$ & $\sigma_{V_{B}}(\mathrm{~km} / \mathrm{s})$ \\
\hline Hough (1911) & -0.661 & 1.27 & 2.01 \\
Lunt (1918)* & -0.02 & 0.833 & 1.26 \\
Campbell \& Moore (1928) & +1.27 & 1.54 & 1.53 \\
Jones (1928) & +0.610 & 0.718 & 1.11 \\
Wesselink (1953) & +3.04 & 0.943 & 0.763 \\
Archer (1957) & +1.70 & 1.10 & 2.57 \\
Jones \& Fisher (1984) & +0.60 & $0.568^{* *}$ & $0.568^{* *}$ \\
\hline \hline
\end{tabular}

The orbit based on all visual and spectroscopic observations is given in columns 4 and 5 of Table 1 and plotted on Fig. 1. It confirms our previous solution. As one expects by adding some new radial velocities only, the visual parameters as well as the parallax remain unchanged.

Though the correlation of $\kappa$ with $V_{0}$ is still very high $(+0.976)$, the improvement of the precision on both parameters is significant. The precision of $\kappa$ is almost three times better than the first estimate. That improvement has 
Spectroscopic orbit

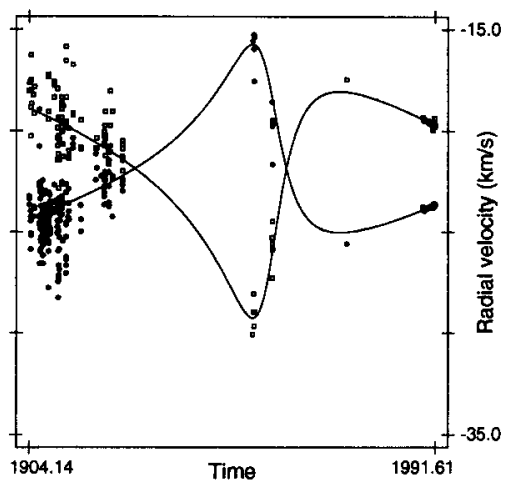

Visual orbit

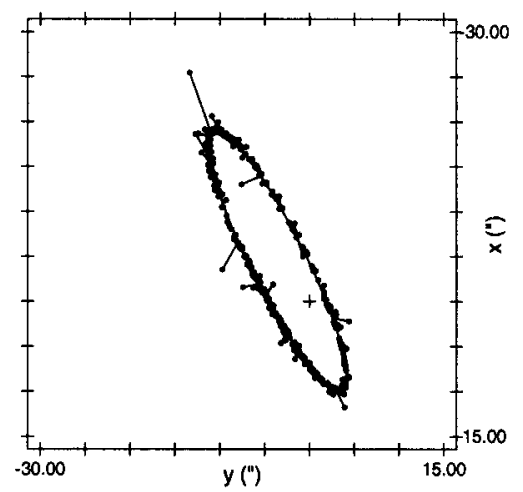

Figure 1. Plot of the orbit corresponding to the best adjustment of all visual and spectroscopic data

noticeable consequences on the individual masses: they are now precise (and, hopefully, accurate) to almost eight per cent.

One could be puzzled noticing that, whereas so many radial velocities have been added, the standard deviation of $\kappa$ is divided by three only. We have indeed added many points but most of them are of rather poor quality (Table 2 ). Actually one would obtain almost the same result by keeping just the four most recent groups (Table 3 ).

Table 3. Value $\kappa$ based on different radial velocity sets of increasing cardinal: sets are added downward

\begin{tabular}{lccc}
\hline Reference & $\kappa$ & $M_{A}\left(M_{\odot}\right)$ & $M_{B}\left(M_{\odot}\right)$ \\
\hline Murdoch et al. (1993) & $0.45 \pm 0.036$ & $1.2 \pm 0.078$ & $1.0 \pm 0.076$ \\
Jones \& Fisher (1984) & $0.45 \pm 0.035$ & $1.2 \pm 0.075$ & $1.0 \pm 0.074$ \\
Archer (1957) & $0.418 \pm 0.026$ & $1.2 \pm 0.057$ & $0.9 \pm 0.055$ \\
Wesselink (1953) & $0.45 \pm 0.013$ & $1.17 \pm 0.032$ & $0.96 \pm 0.031$ \\
Jones (1928) & $0.45 \pm 0.013$ & $1.17 \pm 0.031$ & $0.96 \pm 0.030$ \\
Campbell \& Moore (1928) & $0.45 \pm 0.013$ & $1.16 \pm 0.032$ & $0.97 \pm 0.031$ \\
Lunt (1918) & $0.45 \pm 0.013$ & $1.16 \pm 0.031$ & $0.97 \pm 0.030$ \\
Hough (1911) & $0.45 \pm 0.013$ & $1.16 \pm 0.031$ & $0.97 \pm 0.030$ \\
\hline \hline
\end{tabular}

\section{Hipparcos' results}

The way $\alpha$ Cen has been considered in the reduction process of the Hipparcos (ESA 1997) observations is maybe questionable. Owing to the slow apparent orbital motion of B between 1989.9 and 1993.2, both components have been reduced using the default single star model. In most of the reduction, the two 
stars were not considered as being the members of a binary system. The only exception was the assumption of the uniqueness of the parallax.

That has yielded a solution for both components qualified as uncertain (sic). Multiple solutions are proposed. The adopted parallax, $742 \pm 1.42 \mathrm{mas}$, is a little bit off with respect to our estimate.

\section{Conclusions}

114 years after Downing's request (Glaisher \& Knobel 1884), we propose the first simultaneous spectroscopic-visual orbit of $\alpha$ Cen. The radial velocities of Murdoch et al. (1993) provide a suitable framework to which former observations can be added thus allowing a reliable determination of the mass ratio. The value we obtain is in perfect agreement with the astrometric one (Kamper \& Wesselink 1978). That simultaneous adjustment also yields upward revisions of the distance to the system and of the individual masses of the components (the latter being just a consequence of the former).

Acknowledgments. I thank R.F. Griffin for his valuable help concerning some old bibliographic references (e.g., Glaisher \& Knobel 1884) and also for his careful reading of an early version of this paper.

\section{References}

Archer, S. 1957, MNRAS, 117, 641

Batten, A.H., Fletcher, J.M., \& MacCarthy, D.G. 1989, Publ. Dom. Ap. Obs., 17,1

Campbell, W.W., \& Moore, J.H. 1928, Publ. of the Lick Observatory, 16, 1

Demarque, P., Guenther, D.B., \& van Altena, W.F. 1986, ApJ, 300, 773

ESA, 1997, The Hipparcos and Tycho Catalogues, ESA SP-1200

Glaisher, J.W.L., \& Knobel, E.B. 1884, Observatory, 7, 29

Heintz, W.D. 1958, Veröff. Münch, 5, 100

Heintz, W.D. 1982, Observatory, 102, 42

Hough, S.S. 1911, Annals of the Cape Observatory, 10, 1

Isobe, T., Feigelson, E.D., Akritas, M.G., \& Babu, G.J. 1990, ApJ, 364, 104

Jones, D.H.P., \& Fisher, J.L. 1984, A\&AS, 56, 449

Jones, H.S. 1928, Annals of the Cape Observatory, 10, 95

Kamper, K.W., \& Wesselink, A.J. 1978, AJ, 83, 1653

Lohse, P. 1910, Potsdam Publ., 20, 105

Lunt, J. 1918, ApJ, 48, 182

Murdoch, K., \& Hearnshaw, J.B. 1993, Observatory, 113, 79

Murdoch, K.A., Hearnshaw, J.B., \& Clark, M. 1993, ApJ, 413, 349

Neuforge, C., Pourbaix, D., Noels, A., \& Scuflaire, R. 1999, these Proceedings

Pourbaix, D. 1998, A\&AS, 131, 377

Roberts, A.W. 1893, Astron. Nachr., 133, 105 
Roberts, A.W. 1895, Astron. Nachr., 139, 7

Wesselink, A.J. 1953, MNRAS, 113, 505

Wright, W.H. 1904, ApJ, 20, 140

\section{Discussion}

Hearnshaw: Our mass ratio (Murdoch \& Hearnshaw 1993) was based on onlt 2.5 years of data, so it was indeed a preliminary estimate. Your masses do seem surprisingly high. Do your models include the high metallicity ( $\sim$ twice solar) found from spectral analysis?

Pourbaix: I just wanted to show that, from the same data as you, one can derive a mass ratio closer to the astrometric one, just by using a different adjustment procedure. The masses we obtained are dynamical masses, and they do not require any assumption about the underlying physics. Neuforge et al. (1999) found models for A and B that fit our masses, as well as the other spectroscopic results (surface gravity, metallicity) quite well.

Soderblom: A comment on radial velocities for the $\alpha$ Centauri system - you show almost no data for the 1980 's, but during that time $\alpha$ Cen was observed many times with the CAT + CES at ESO. I suggest you inquire of European observers to see if they have data to fill in that period.

Pourbaix: Thank you very much for that information. 